\title{
Estimated Pulse Height Spectrum with Pulse Pile-Up Correction for Neutron Monitor of Mexico City
}

\author{
Rocío García $^{* a}$, J.F.Valdés-Galicia ${ }^{a}$, Marcos Anzorena $^{a}$, Octavio Musalem $^{a}$, \\ Alejandro Hurtado ${ }^{a}$, Ernesto Ortiz $^{b}$, L.X.González ${ }^{c}$. \\ ${ }^{a}$ Instituto de Geofísica, Universidad Nacional Autónoma de México,Coyoacán,CDMX, 04510, \\ México. \\ ${ }^{b}$ Instituto de Ciencias Físicas, Universidad Nacional Autónoma de México, Cuernavaca, \\ Morelos,62210, MEXICO. \\ 'SCiESMEX, Instituto de Geofísica, Unidad Michoacán, Universidad Nacional Autónoma de \\ México, Michoacán Morelia, 58190, MEXICO. \\ E-mail: rocio@geofisica.unam.mx
}

\begin{abstract}
The operating principles of Neutron Monitors are nuclear reactions within the proportional counters. The output signal of these is an electric pulse for every secondary cosmic ray particle that interacts with the detector gas. The amplitude of the pulse signal reflects the amount of charge generated on each individual interaction. The estimated pulse height distribution provides an estimate of the energy deposited. The random nature of the cosmic radiation, in addition to the operating characteristics of both the detector and the associated electronic system, lead to a phenomenon called pulse pile-up. The effect of the pulse pile-up on the recorded pulse height distribution, may be seen in the added wings in the energy peaks of the distribution. This reduces the energy resolution of the instrument.

In this work, we describe an algorithm that takes advantage of digital signal processing techniques with the purpose of calculating the pulse amplitude distribution reducing the distortion caused by pulse pileup. The algorithm was written in python, using numpy and scipy libraries. The results of applying the algorithm to the neutron monitor operating in Mexico City are presented.
\end{abstract}

35th International Cosmic Ray Conference

10-20 July, 2017

Bexco, Busan, Korea

* Speaker. 


\section{Introduction}

The Neutron Monitor (NM) currently in use in many cosmic ray stations around the world is sensitive to primary cosmic rays on the energy range 0.5 to $40 \mathrm{GeV}$. NMs are designed to register cosmic ray intensity using the pulse mode operation technique; every pulse represents a radiation event, carrying information on all the charge released in the detector. This information is contained in the pulse height of each event. The statistics of the NM is very good, a typical 6-NM64 is capable of counting rates up to $1 \times 10^{6}$ events $/ \mathrm{h}$.

A schematic diagram of a 6-NM64 neutron monitor is shown in figure 1. One such detector has been in continuous operation in Mexico City since 1990[1]. Incoming secondary cosmic rays cross a low density polyethylene reflector, without significant energy loss. Reflector bounces back neutrons produced inside the monitor towards proportional counters for their detection and absorbs low energy neutron external background. Polyethylene tubes and lead rings surround proportional counters (PRC) as moderator/producer to enhance detection probability. As the neutrons produced in the polyethylene tubes are in the thermal energy range, with large cross sections for Boron, the reaction produces Lithium and an alpha particle that ionizes the $B F_{3}$ gas of the PRC to produce the desired pulses in the anode.

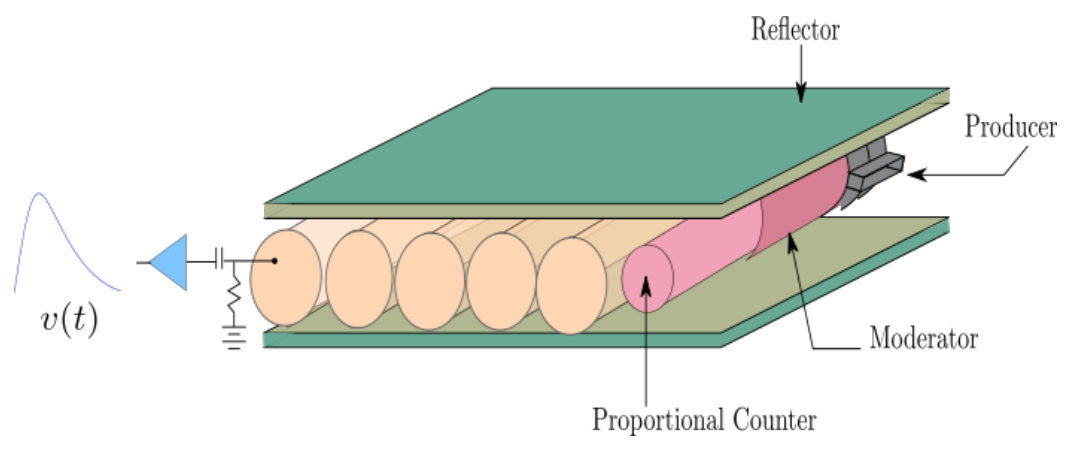

Figure 1: Schematic diagram of the Neutron Monitor

A finite period of time is necessary to collect the charge produced in the anode. This signal is amplified and processed to derive information about the incoming radiation.

\section{Pulse Height Distribution and pile-up phenomena}

Pulse height distributions from radiation sources are structured in general; ridges and valleys represent the energy distribution of the source, the interactions of quanta with the detector and its electrical properties. Therefore, the pulse height distribution provides a diagnostic of the detector, using energy resolution as an indicator.

Exposed to a monoenergetic low speed neutron flux, a $B F_{3}$ proportional counter has a very definite and simple pulse height distribution (Figure 2) with an energy resolution of around $8.5 \%$ [2]. No pulse pile-up is appreciated.

However, the random nature of the cosmic ray flux, together with the characteristics of the PRC, pulse pile-up is possible when events occur within a small time window ( $\sim 20 \mu \mathrm{s})$. Two types of pile-up signals are significant: Tail pile-up and peak pile-up. Tail pile-up occurs when a second 


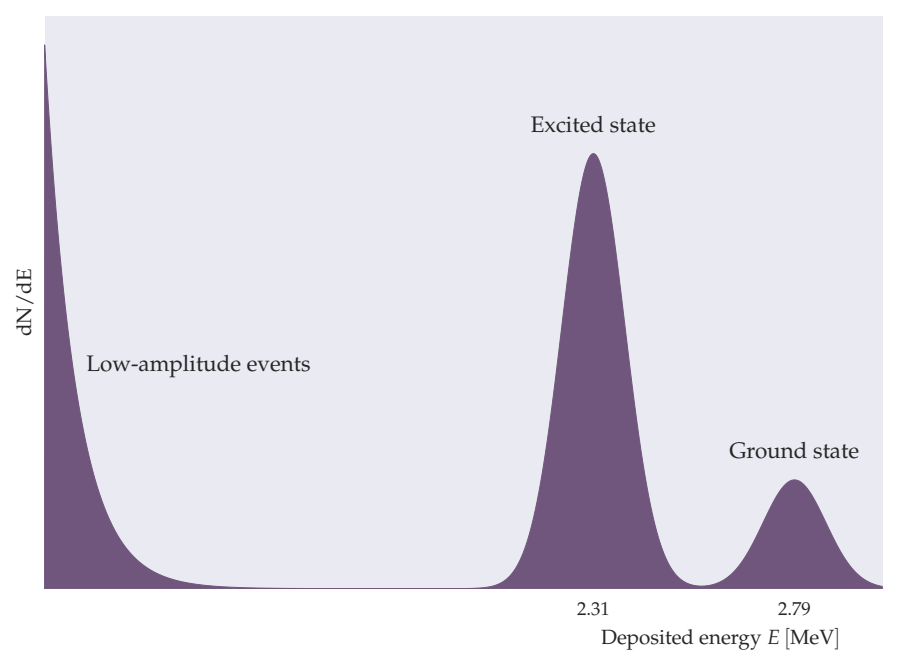

Figure 2: Energy Spectrum obtained from a $B F_{3}$ proportional counter. When a slow neutron interacts with boron-10 it produces an $\alpha$-particle with two possible energies $(2.31 \mathrm{MeV}$ and $2.79 \mathrm{MeV}$ )

event is superimposed after the peak of the previous. Height spectra may be skewed to higher or lower values. Peak pile-up arises when two events are very close in time; therefore only one pulse is recorded, affecting pulse height distribution and reducing the detector's efficiency.

In this work we develop a digital processing technique to minimize pulse height distortion by pile-up phenomena, and reach a more realistic pulse height distribution

\section{The Different Techniques in use}

Pulse width shortening is one of the most common techniques in use to reduce pile-up effects. Spectroscopy experiments employ linear filters to attenuate slow decaying component signals[3], [4]. A very profound knowledge of the signal is necessary in this case; otherwise it may lead to detection of false pulses and the degradation of the signal to noise ratio.

Considering only well defined pulses to construct the pulse height spectrum may be used at very low counting rates. However, this technique becomes ineffective as the counting rate increases.

Modeling the detector signal as the sum of an unknown number of randomly arriving radiation events, with a defined pulse shape but unknown energy distribution have been tried recently[5]. Nevertheless the complexities involved in modeling the great number of physical processes involved in the radiation-matter interaction limits considerably this approach.

The NM in Mexico City does not receive a very high particle flux. Therefore we decided to use an offline peak detection algorithm to reduce pile-up effects. The algorithm uses the digital output of the preamplifiers, without signal preconditioning.

\section{Methodology}

Neutron monitors operate in pulse counting mode, therefore in order to obtain the pulse height distribution we must digitize first the output signal from the PRCs. Current pulses from the PRCs 
are integrated and converted into voltage signals. After amplification $(G=45)$, the signal pass through an integral discriminator with a threshold of $300 \mathrm{mV}[2]$. We digitize the signal, after amplification stage and before discrimination, using a digital oscilloscope (Tektronix MSO2024). We will call this discrete time signal $x[n]$. Each window of data is $1 \mathrm{~ms}$ length. Figure 3 shows and example of one data window from $x[n]$

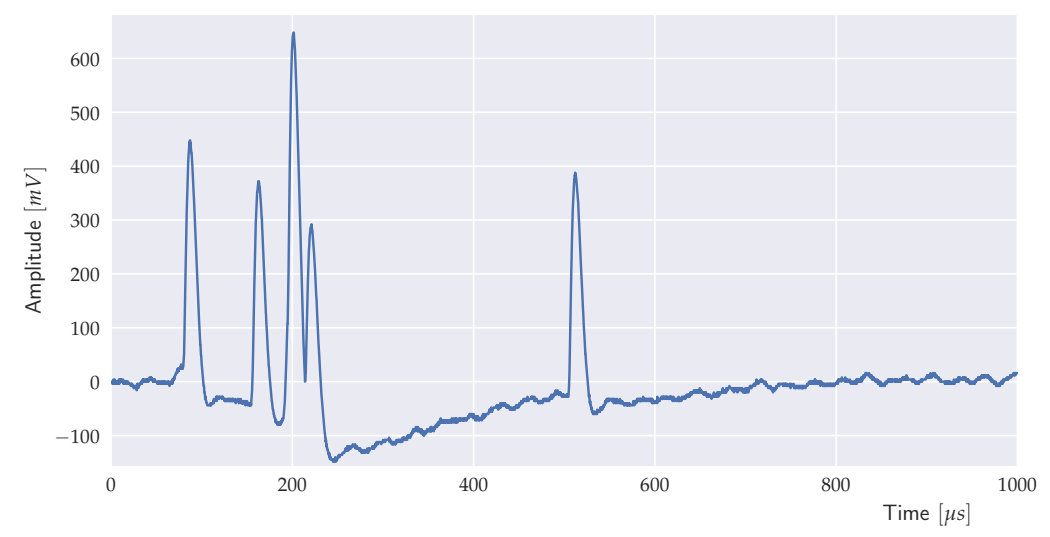

Figure 3: Registered pulse waveform from one proportional counter of the Neutron Monitor in Mexico City. The effect of pulse pile-up is clearly seen in this figure

The software produced is based on the wavelet transform using ridge line detection to finally construct histograms. We adapt the algorithm in[6] to process the signals of the NM. The advantages of the technique are the inexistence of signal conditioning, reduction of noise and tail pile-up. The software is written in Phyton.

\subsection{Continuous wavelet transform}

The continuous wavelet transform (CWT) may be defined as the convolution of a signal $x(t)$ with a set of functions $\psi_{s, v}(t)$ called wavelet family, which are a scaled and translated version of the wavelet function $\psi(t)[7]$ :

$$
C W T=\frac{1}{\sqrt{s}} \int x(t) \psi^{*}\left(\frac{t-v}{s}\right) d t
$$

where $*$ denotes complex conjugate and $\frac{1}{\sqrt{s}}$ is a normalization factor. The CWT is the mapping of the signal to the time-frequency space, providing information about frequency content of the signal and its time occurrence.

Using the convolution theorem, we construct the CWT by multiplying the Discrete Fourier transforms (DFT) of the discrete time signal $x[n]$ and the wavelet function and then taking the inverse transform. We use the second derivative of a Gaussian (mexican-hat) as the wavelet function because it is a real function and has similar shape to the pulses from the PRCs. The DFT of the Mexican hat function is well known[7]

The result of the transformation is a matrix of wavelet coefficients $C_{\psi}$, representing a measure of the similarity between both signals. Large $C_{\psi}$ represent a wavelet function closer to the detector signal. Figure4 is an example of data from one PRC of the NM, mapped to the time-frequency space. Peaks in the data are reflected over the scales (frequencies) band. 

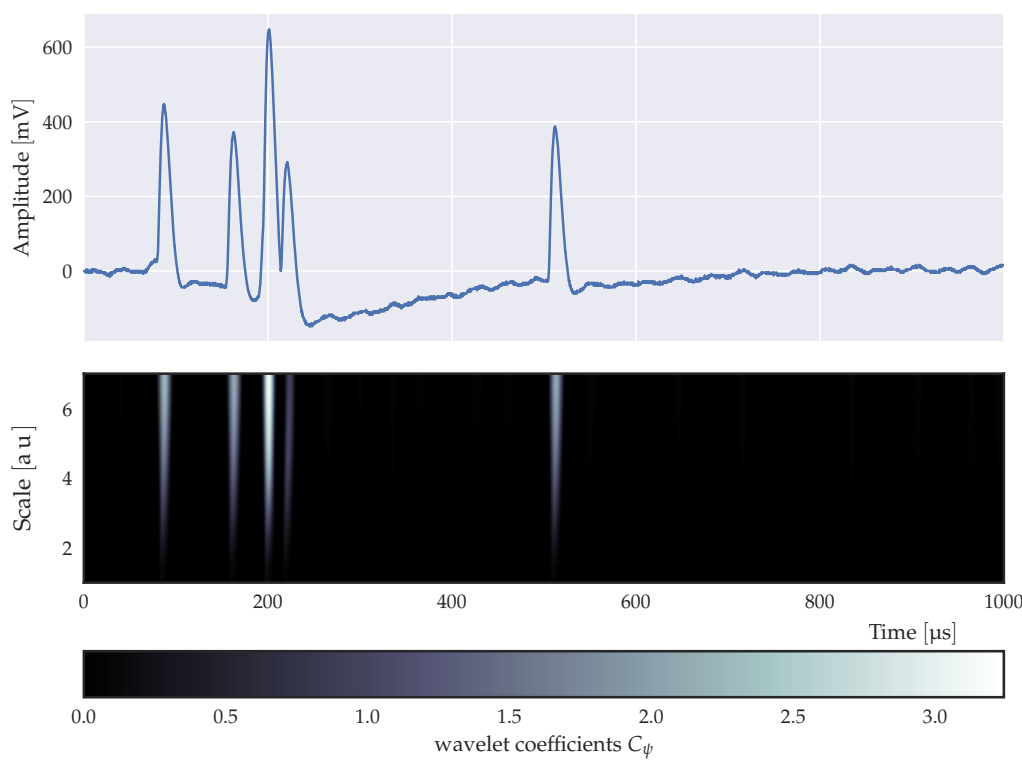

Figure 4: Continuous wavelet transform for cosmic ray events detected with NM. The top figure shows the $x[n]$ signal and the bottom figure shows the time-frequency space. The colors in the figure represent a scale for wavelet coefficients values

\subsection{Ridge line detection}

Once we have calculated the $C_{\psi}$ matrix we separate cosmic rays real events from noise. Real events cover a wide range of scales(frequencies); noise peaks cover a narrower range. The procedure may be summarized as follows:

- Identify the largest coefficients on each scale.

- Search for largest coefficients in adjacent scales. Link them to form ridge lines.

- Ridge lines associated to real signals must satisfy:

- Signal to noise ratio (SNR) of the ridge line must be higher than a given threshold.

- The length of the ridge must be larger than established minimum

Analyzing signals from PCs we determined a noise level of $21 \mathrm{mV}$, very low compared with pulse amplitude $400 \mathrm{mV}$. The analysis lead to establish a lower threshold for real peaks at $100 \mathrm{mV}$.

Not all pile-up cases may be identified. Wavelet coefficients $>4.0$ on scales larger than 5 are due to pile-up of a single cosmic ray. The maximum separation between single events must be $<5 \mu$ s to be considered as peak pile-up. This corresponds to the falling time of the pulse. The algorithm is able to detect the single events only if they are recorded on smaller scales(see Figure5)

Tail pile-up is eliminated directly using the wavelet transform, because convolution with the wavelet function acts as as band-pass filter on the signal, attenuating the the slow recovering phase from undershot. 

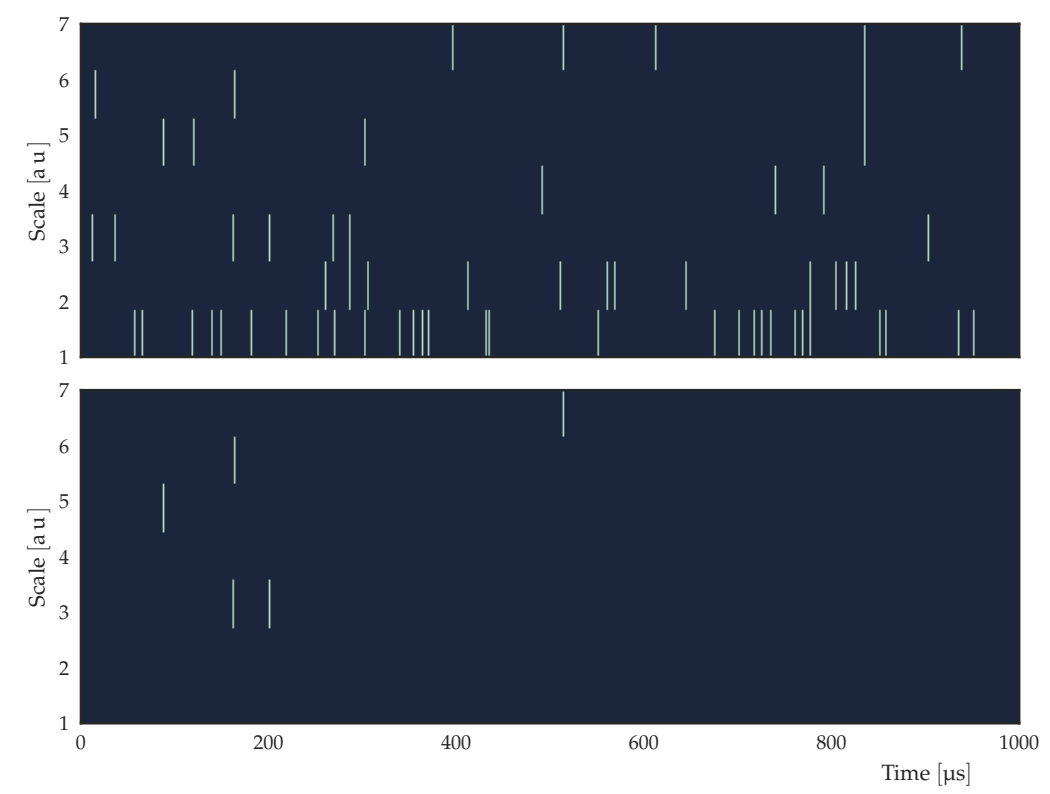

Figure 5: The top panel shows the ridge lines detected by the algorithm and the bottom panel shows the filtered real cosmic rays events

\subsection{Pulse Height Distribution}

To estimate the pulse height distribution from the PRC we construct an histogram using the wavelet coefficients. As pulse height is proportional to the energy deposited, the coefficients are good estimators of energy. Hence, if the wavelet function is normalized on every scale, CWT coefficients mainly variate according to pulse amplitude.

The energy resolution $E_{R}$ for each PRC may be estimated from:

$$
E_{R}=\frac{F W H M}{H_{0}}
$$

Where FWHM is the full width at half maximum of the main peak and $H_{0}$ is the average pulse height. We fit the histogram to a Gaussian distribution and validate using the $R^{2}$ coefficient of determination.

\section{Results and Discussion}

We will refer to the six PCs in the NM as $A 1, A 2, B 1, B 2, C 1$ and $C 2$. Figure6 is an example of the pulse height distribution of $A 2$ counter. Distributions are very similar for $B 2, C 1$ and $C 2$. They follow the expected shape, with peaks representative of ground state and excited Lithium; in all of them the ground state peak (right) is less than $10 \%$ of the excited state (left) peak.

Counters $A 1$ and $B 1$ show some distortions in the pulse shape distributions that are due to malfunctioning of the preamplifiers. We have replaced the corresponding electronics and, at the time of writing this paper, we are in the process of rebuilding pulse height distributions for these PRCs. 


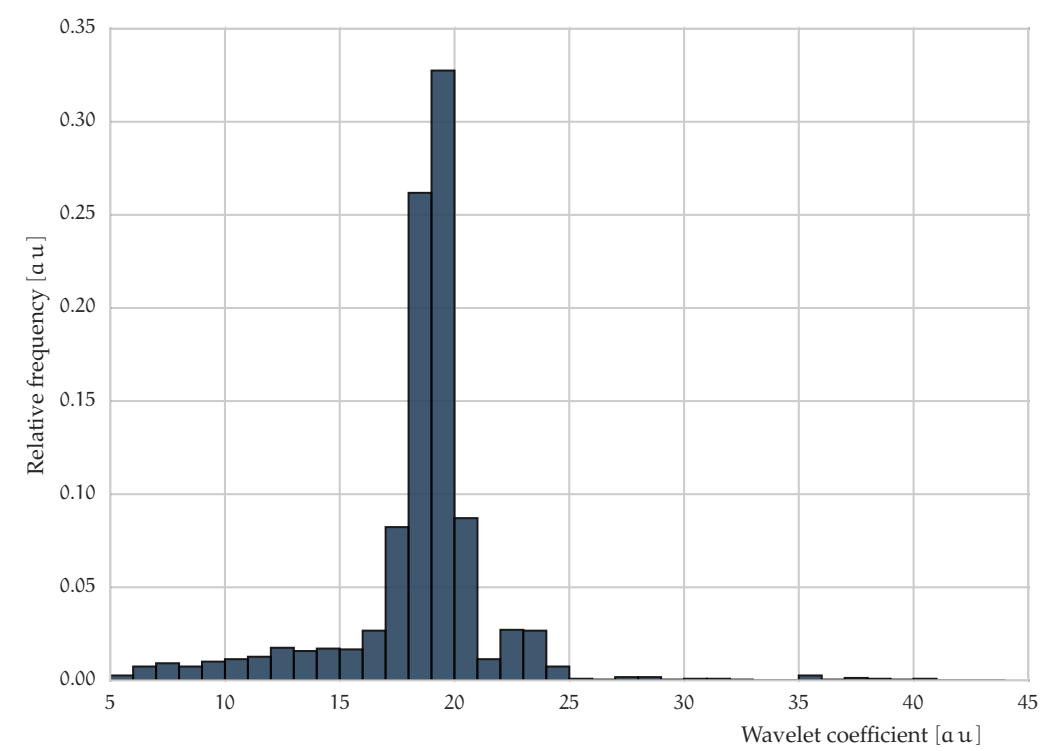

Figure 6: Wavelet Coefficients Distribution for proportional counter $A 2$

\begin{tabular}{ccccc}
\hline & \multicolumn{2}{c}{ Wavelet coefficient } & \multicolumn{2}{c}{ Local maxima } \\
\cline { 2 - 5 } Counter & $E_{\phi}[\%]$ & $R^{2}$ & $E_{L M}[\%]$ & $R^{2}$ \\
$A 1$ & none & --- & none & --- \\
$A 2$ & 10.80 & 0.98 & 9.44 & 0.86 \\
$B 1$ & none & --- & none & --- \\
$B 2$ & 10.40 & 0.95 & 10.79 & 0.83 \\
$C 1$ & 15.00 & 0.99 & 17.42 & 0.92 \\
$C 2$ & 09.56 & 0.98 & 9.31 & 0.81 \\
\hline
\end{tabular}

Table 1: Evaluation of the resolution by wavelet transform $E_{\phi}$ and local maxima algorithms $E_{L M}$.

The estimated energy resolution $E_{R}$ for the four PRCs are shown in table1. As stated above, a $B P 28$ counter under a mono-energetic slow neutron source has an energy resolution of $8.5 \%$. For a NM64 standard is required a maximum resolution of $20 \%$ for PRCs[2]. Table 1 shows that all PRCs satisfy this requirement, which guarantees the reliable operation of the Mexico City NM.

Very high amplitude $(>400 \mathrm{mV}$ ) pile-up pulses produced by cosmic ray events closely spaced in time are not resolved by the algorithm presented here. Nevertheless, less than $1 \%$ of the detected events correspond to this case, therefore these signals may be eliminated from the dataset.

To evaluate this, we estimated the spectrum using a different method based on local maxima and compare the results with the wavelet analysis. The local maxima method uses finite differences in search for inflection points. Figure 7 shows the pulse height distribution from each the four PRCs that show manageable results, using the local maxima approach. It is clear from the figure that there is a greater degree of distortion on the distributions. This is because, even though local maxima may correctly detect the positions of the maxima, the value of the pulse amplitude is affected by tail pile-up. 


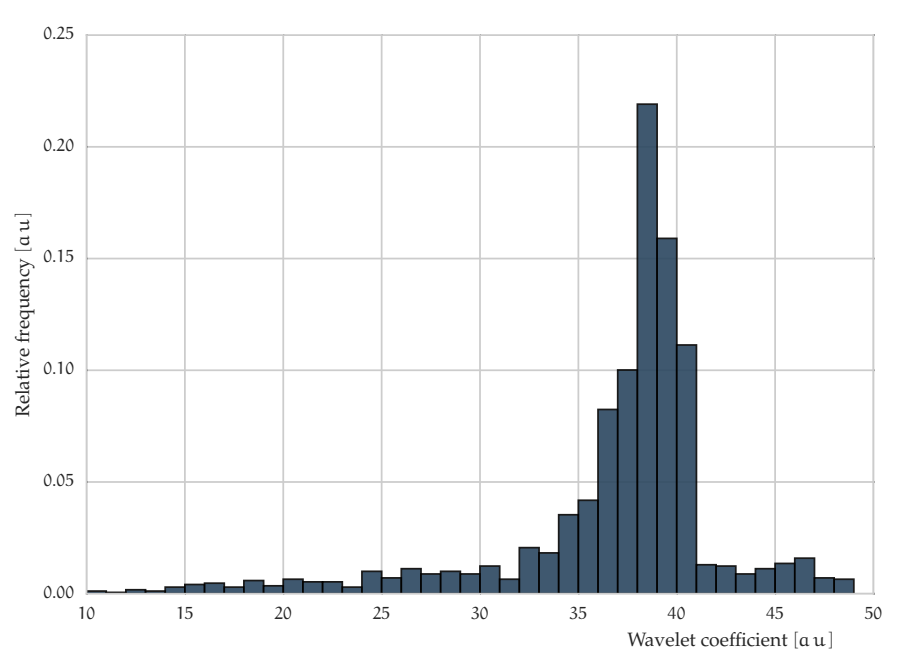

Figure 7: Pulse High Distribution using local maxima method for proportional counter $A 2$

\section{Summary}

We produced an algorithm that successfully detected pulse pile-up in the NM of Mexico City and was able to clean the output signals from these effects. A careful evaluation of the phenomenon led us to the conclusion that around $3 \%$ of the output data are affected by pile-up. The algorithm allowed us to evaluate the energy resolution of the NM to be around $16 \%$.

\section{Acknowledgments}

This work is supported by UNAM-PAPIIT-IN104115 and CONACyT-180727T. We want to thank Mexican Physics Society and LOC of the 35th ICRC for the funding to attend conference.

\section{References}

[1] Instituto de Geofísica, UNAM, "Mexico city cosmic ray observatory: Real time cosmic preliminary data." http: // www. cosmicrays. unam.mx/, 2017.

[2] H. Carmichael, IQSY Instruction Manual, Tech. Rep. 7, Deep river, Canada, 1964.

[3] A.Messai, et. al., Digital signal processing for optimal resolution in gamma ray spectroscopy, in Instrumentation for Digital Nuclear Spectroscopy, pp. 261-264, The IAEA Technical Meeting Held in Vienna, Australia, 2010.

[4] Valentin T. Jordanov, et. al., Digital techniques for real-time pulse shaping in radiation measurements, Nuclear Instruments and Methods in Physics Research A 353 (1994) 261-264.

[5] Paul A. B. Scoullar, et. al. , Maximum Likelihood Estimation Techniques for High Rate, High Throughput Digital Pulse Processing, 2008.

[6] P. Du, et al., Improved peak detection in mass spectrum by incorporating continuous wavelet transform-based pattern matching, Bioinformatics 22:17 (2006) 2059-2065.

[7] S. Mallat, A wavelet tour of signal processing: The sparse way. Academic press, United States, 3rd ed., 2008. 